\title{
How hard is a colloidal "hard-sphere" interaction?
}

\author{
G. Bryant ${ }^{1, *}$ S. R. Williams, ${ }^{2}$ L. Qian, ${ }^{3}$ I. K. Snook, ${ }^{1}$ E. Perez,${ }^{3}$ and F. Pincet ${ }^{3}$ \\ ${ }^{1}$ Department of Applied Physics, Royal Melbourne Institute of Technology, GPO Box 2476V, Melbourne, 3001 Australia \\ ${ }^{2}$ Van 't Hoff Laboratory for Physical and Colloidal Chemistry, Debye Institute, University of Utrecht, Padualaan 8 , \\ 3508 TB Utrecht, The Netherlands \\ ${ }^{3}$ Laboratoire de Physique Statistique de l'Ecole Normale Supérieure, associé aux Univerités Paris 6 et Paris 7 , \\ 75231 Paris Cedex 05, France
}

(Received 21 October 2002; published 30 December 2002)

\begin{abstract}
Poly-12-hydroxystearic acid (PHSA) is widely used as a coating on colloidal spheres to provide a "hardsphere-type" interaction. These hard spheres have been widely used in fundamental studies of nucleation, crystallization, and glass formation. Most authors describe the interaction as "nearly" hard sphere. In this paper we directly measure this interaction, using layers of PHSA adsorbed onto mica sheets in a surfaces force apparatus. We find that the layers, in appropriate solvents, have no long-range interaction. When the solvent is decahydronaphthalene (decalin), the repulsion rises from zero to the maximum measurable over a distance range of 15-20 nm. The data is converted to equivalent forces between spheres of different diameters, and modeled using a hard core potential. Using zeroth-order perturbation theory and computer simulation, we demonstrate that the equation of state does not deviate from that of a perfect hard-sphere system under any relevant experimental conditions.
\end{abstract}

DOI: 10.1103/PhysRevE.66.060501

PACS number(s): 64.70.Dv, 82.70.Dd

\section{INTRODUCTION}

Sterically stabilized colloidal particles have been used as a model for hard spheres for a number of years by many different groups (e.g., Refs. [1-5]). They represent an excellent model system for studying crystallization and vitrification at a fundamental level. It has been assumed that these experimental systems behave as perfect hard spheres, and indeed these systems have been assigned a reference status for the glass transition [6].

Recent progress in classical nucleation theory has resulted in the successful calculation of the absolute nucleation rate using Monte Carlo techniques [7,8]. Nucleation rates have been predicted to depend sensitively on the interaction softness [8], and they have also been compared to results obtained from confocal microscopy experiments on dispersions of charge stabilized spheres [9]. However, an incomplete characterization of the effective pair potential did not allow for a rigorous testing of the theory. Clearly, a detailed knowledge of the pair interactions for model colloidal systems is of the utmost importance. Here we address the effect of particle softness for sterically stabilized colloidal spheres without the added complication of polydispersity.

There have been several tests of the validity of the hardsphere assumption for poly-12-hydroxystearic acid (PHSA) coated particles. Sedimentation studies $[1,10]$ show that the experimental miscibility gap (i.e., the difference between the melting and freezing volume fractions) agrees with theoretical predictions, which means that freezing occurs at the effective hard-sphere volume fraction of 0.494 and melting occurs at 0.545, within experimental errors. However, this is a very imprecise test, as the miscibility gap is insensitive to the detailed shape of the interaction [11]. In addition, this

*Electronic address: gary.bryant@ rmit.edu.au measure only tests the validity of the hard-sphere model at the phase boundaries. There is also the further complication that polydispersity can affect the miscibility gap [12], and this parameter is often poorly characterized. Viscosity measurements of dilute suspensions [10] can be used to determine the effective hard-sphere radius, but it would not be expected that such dilute suspensions would be affected by particle softness to the same extent as the high density metastable or glassy phases found in phase transition studies. The force between PHSA layers has been measured [13], but here comparisons were made with rheological properties, and this work did not address the nature of the hard-sphere interaction.

So while there is good evidence that these sterically stabilized particles behave as "nearly" hard-spheres, the "nearly" needs to be better characterized. In this paper we perform experiments to directly measure the interactions between the layers of PHSA, which comprise the "hardsphere" layer around the particles. We fit a hard core function to the experimental data for a range of relevant values of particle radius, and using this we obtain the equation of state from Monte Carlo simulations. The effective hard-sphere diameter is fixed using zeroth-order hard-sphere perturbation theory. We apply this to particles of different diameters over the colloidal range of particle sizes, and determine how close the particles are to being perfect hard spheres.

\section{THEORY AND METHODS}

\section{A. Theory}

Simulations of the phase behavior of spheres with purely repulsive potentials usually either consist of perfect hard spheres, or a continuous soft-sphere potential $U(r)$ with the form

$$
U=\varepsilon(\sigma / r)^{n},
$$


where $\varepsilon$ is the strength of the interaction, $r$ is the center-tocenter separation, $\sigma$ is the range of the interaction, and $n$ is the repulsive index.

In order to model the experimental condition of a hard core with a soft potential, we choose the following form for a hard-core potential:

$$
\begin{gathered}
u(r)=\infty, \quad r<\sigma_{h}, \\
u(r)=\frac{\varepsilon}{\left[\left(\frac{r}{\sigma_{h}}\right)^{n}-1\right]}, \quad r>\sigma_{h} .
\end{gathered}
$$

This form represents an infinitely hard core with a diameter $\sigma_{h}$ surrounded by a "soft" potential, characterized by the repulsive index $n$. This equation has enough independently free parameters to allow the experimental force measurements to be accurately fitted.

\section{B. Zeroth-order perturbation theory}

In order to compare systems with potentials $u(r)$ of the form given by Eqs. (1) and (2) to hard-sphere systems, we need to determine an effective hard-sphere diameter. The effective hard-sphere diameter $\sigma_{B H}$ may be obtained by the method of Barker and Henderson [14], which is derived by annulling the first-order difference between the Helmholtz free energy of the hard-sphere system and the system with pair potential $u(r)$. This gives [14]

$$
\sigma_{B H}=\int_{0}^{\infty} 1-e^{-u(r) / k T} d r .
$$

\section{Simulation details}

Simulations were performed using molecular dynamics and Monte Carlo techniques. The equation of state data for the soft-sphere potential, Eq. (1), was obtained by the use of molecular dynamics in the canonical ensemble employing the Verlet algorithm [15] with a Gaussian thermostat [16]. Equation of state data for the hard-core potential, Eq. (2), was obtained by Monte Carlo simulations in the isothermalisobaric ensemble [15]. The choice between the two simulation methods was made for convenience.

\section{Comparing hard-core force and SFA measurements}

Differentiating the hard-core potential [Eq. (2)] with respect to $r$ gives the force

$$
F(r)=\frac{\frac{\varepsilon n}{\sigma_{h}}\left(\frac{r}{\sigma_{h}}\right)^{n-1}}{\left[\left(\frac{r}{\sigma_{h}}\right)^{n}-1\right]^{2}} .
$$

The surface force apparatus (SFA) measures the force as a function of surface-to-surface separation $d$ between two curved surfaces in a crossed cylinder configuration. This force can be related to the energy per unit area between parallel plates by $E_{\|}(d)$ by, e.g., Ref. [17]:

$$
\frac{F_{S F A}(d)}{R}=2 \pi E_{\|}(d)
$$

where $R$ is the radius of curvature of the surfaces in the SFA $(\approx 2 \mathrm{~cm})$.

But $E_{\|}(d)$ may also be related to the force between two spheres of diameter $\sigma_{c}$ by, e.g., Ref. [17]:

$$
\frac{F(d)}{\sigma_{c} / 2}=\pi E_{\|}(d) \text {. }
$$

Thus the force between two spheres of diameter $\sigma_{c}$ may be related to the SFA measurements. In order to compare with the hard-core model, we write it as a function of center-tocenter separation $r$ by the transformation $r=d+\sigma_{c}$, which is valid for $r>\sigma_{c}$. The force between two spheres can then be determined by the expression

$$
F(r)=\frac{\sigma_{c}}{4 R} F_{S F A}(r)
$$

In general, $\sigma_{c}$ will be different compared to $\sigma_{h}$ [Eq. (2)]. In the SFA, the zero position is defined as contact between the mica sheets. In the case of colloidal particles, this is equivalent to contact between the particle cores (i.e., it does not include the thickness of the PHSA layer). So $\sigma_{c}$ represents the diameter of the particle cores, and $\sigma_{h}$ represents the diameter at which the hard-core potential becomes infinitei.e., the separation at which the PHSA layers are fully interdigitated.

From Eq. (7), it is clear that the equivalent force between pairs of particles with different diameters increases with sphere diameter. Essentially, as the particle diameter increases, a larger area of surface is closer to the neighboring particle, so the strength of the interaction increases.

\section{EXPERIMENT}

The SFA [18] allows the measurement of the force as a function of the distance between two molecularly smooth surfaces separated by a liquid. The force is measured with a cantilever spring $( \pm 1 \mu \mathrm{N})$, and the distance is obtained by multiple beam interferometry $( \pm 0.2 \mathrm{~nm})$. The two surfaces are arranged in a crossed cylinder geometry (radius $R$ ).

Two solvents were used. Decahydronaphthalene (mixed cis and trans, 98\% Janssen), referred to by its commercial name decalin; and $n$-dodecane (Merck, 99\%). The PHSA was from the RMIT department of Applied Physics Colloids Laboratory. All solvents were filtered through a $0.5-\mu \mathrm{m}$ filter before use. The PHSA was dissolved in the appropriate solvent (decalin or dodecane), until no more could be dissolved by simple mixing. This solution was then filtered.

The glass cylindrical lenses with attached mica were placed in the PHSA solution for $15 \mathrm{~min}$ (decalin) or $10 \mathrm{~min}$ (dodecane) in order to allow the PHSA to deposit on the mica. After this, the lenses were removed and rinsed of excess PHSA by shaking for about a minute in pure solvent. 

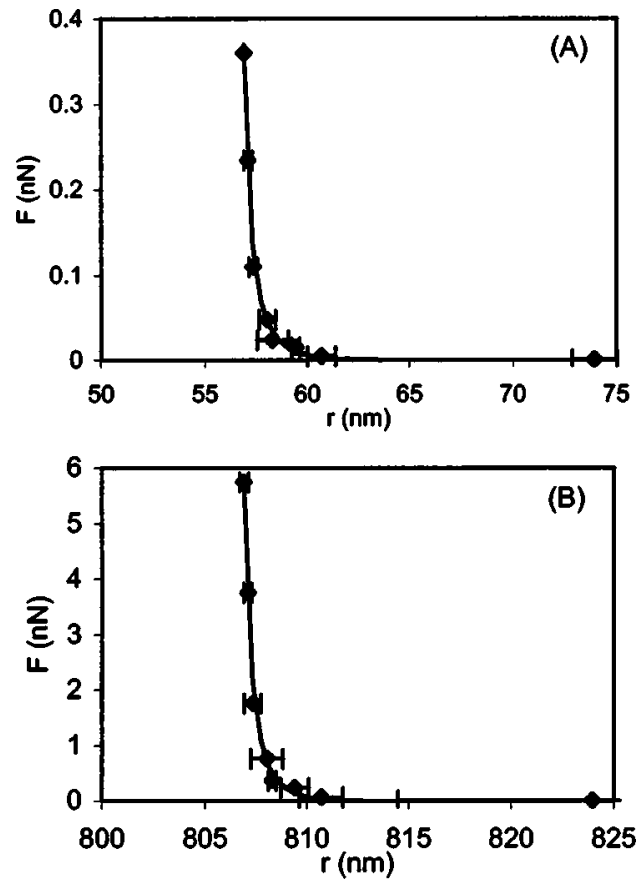

FIG. 1. Force as a function of diameter for spheres with core diameters of (a) $50 \mathrm{~nm}$ and (b) $800 \mathrm{~nm}$. The points represent the experimental data, and the lines are the best fits using Eq. (2). Error bars are the standard deviations of the values from six curves.

They were then transferred to another container of fresh solvent, and allowed to sit for 30 min prior to transferring to the SFA. Once in the SFA, a drop of the solvent $(\sim 50 \mu \mathrm{l})$ was placed between the mica surfaces. The SFA was then sealed and transferred to the experimental chamber. After waiting for approximately $30 \mathrm{~min}$ for equilibration, the experiments were begun. Experiments were conducted at $24^{\circ} \mathrm{C}$.

The forces are measured by cycles of bringing together and separating the surfaces. To test the reproducibility of the measurements for each case studied, two different experiments, i.e., with different pairs of mica surfaces, were performed, and in each experiment, three cycles were performed. No hysteresis was observed within the experimental error. After drift correction, the results for all curves were averaged to yield the final results. The raw SFA results were then converted to an equivalent force between spheres of diameter $\sigma_{c}$ using Eq. (7).

\section{RESULTS AND DISCUSSION}

Figure 1 shows the average data converted to the equivalent force between spheres for core diameters $\sigma_{c}$ of (a) 50 $\mathrm{nm}$ and (b) $800 \mathrm{~nm}$. In both cases the repulsion begins to rise above the background at about $11 \mathrm{~nm}$ from the surface. For the $800-\mathrm{nm}$ particles, however, the force is about 10 times larger than for the 50-nm particles. Although the two fits use the same raw data, there is no universal curve, as both the magnitudes of the force and separation at which it occurs, are dependent on the core diameter chosen [Eq. (7)]. Thus the fits need to be carried out for each core diameter to determine how hard the interaction will be.

The lines in the figure represent the best fits to the data
TABLE I. Fit parameters obtained from fitting the hard-core force [Eq. (2)] to the experimental force data. Equation (5) is used to convert from the SFA measurements to the equivalent force between spheres of diameter $\sigma_{c} . \sigma_{B H}$ is the effective hard sphere diameter, determined by the method of Barker and Henderson (10), Eq. (3). All fits have an $R^{2}$ value of 0.994 .

\begin{tabular}{lcccccc}
\hline \hline $\begin{array}{l}\sigma_{c} \\
(\mathrm{~nm})\end{array}$ & $\begin{array}{c}\sigma_{h} \\
(\mathrm{~nm})\end{array}$ & $n$ & $\begin{array}{c}\varepsilon \\
\left(10^{-21} \mathrm{~J}\right)\end{array}$ & $\begin{array}{c}\varepsilon \\
(k T)\end{array}$ & $\begin{array}{c}\sigma_{1 k T} \\
(\mathrm{~nm})\end{array}$ & $\begin{array}{c}\sigma_{B H} \\
(\mathrm{~nm})\end{array}$ \\
\hline 50 & 56.21 & 46 & 149 & 36 & 60.8 & 61.59 \\
100 & 106.20 & 87 & 296 & 72 & 111.6 & 112.32 \\
200 & 206.20 & 170 & 599 & 146 & 212.3 & 213.07 \\
400 & 406.19 & 363 & 1351 & 329 & 412.7 & 413.39 \\
800 & 806.17 & 772 & 3041 & 739 & 813.0 & 813.71 \\
\hline \hline
\end{tabular}

assuming the hard-core form of the force [Eq. (2)]. The fit parameters for these two diameters are shown in Table I, along with the parameters for particles with diameters between these two extremes. $\sigma_{c}$ is the core diameter of the PMMA particles, and corresponds to the optical core measured by light scattering [19]. In the SFA it corresponds to the bare mica touching. The difference between the core diameter and $\sigma_{h}$ is $6.2 \pm 0.1 \mathrm{~nm}$, so this distance is universal, as would be expected.

The other parameters are much more imprecise from the fits, but the trend is clear. Both the repulsive index $n$ and the strength of the repulsion $\varepsilon$ increase with increasing diameter. Only for the very small particles is the value of $n$ approaching a value small enough that there may be some deviations from perfect hard-sphere behavior. The maximum range of the interaction (i.e., the distance from contact where the force first deviates from the large separation background) is about $11 \mathrm{~nm}$. Between 11 and $15 \mathrm{~nm}$, there are no data points, so there may be some very small interaction out to about $15 \mathrm{~nm}$.

How does this compare with other known facts about the particles? The core diameter of a colloidal particle can be determined optically with a high degree of accuracy $[19,20]$. The PHSA layer on the colloidal particles is estimated, by optical methods, to have a thickness between 8 and $15 \mathrm{~nm}$ (the variation is due to slight differences between different particle preparations and different particle sizes). So, one would assume that the interaction would begin when the PHSA layers begin to overlap, about $8-15 \mathrm{~nm}$, and this is consistent with the 11-15 $\mathrm{nm}$ range measured. The maximum repulsion occurs at $6.2 \mathrm{~nm}$, about half this distance. At this point the PHSA layers are maximally interdigitated, and the force of repulsion becomes exceedingly large.

In Table I the strength $\varepsilon$ is also shown in units of $k T$. Even for the smallest particle diameter the spheres are very hard, as $\varepsilon \sim 36 k T$, high enough that Brownian particles would never come this close together. Also shown in Table I is the calculated core diameter $\sigma_{1 k T}$ when the energy is equal to $1 k T$. This indicates that under normal Brownian conditions, particles would rarely come much closer together than this separation, and that this distance approximates an "effective" hard-sphere diameter.

This analysis shows that the particles should behave as perfect hard spheres under most conditions. However, to fur- 


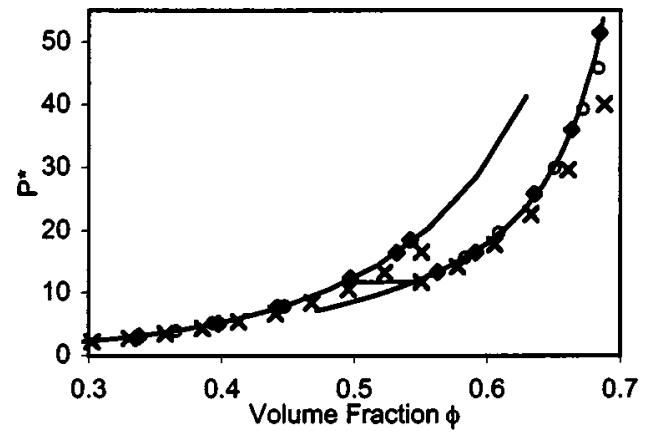

FIG. 2. Comparison of the known hard-sphere equation of state (solid lines) with the simulations. The pressure is in dimensionless units such that $P^{*}=P \sigma_{B H}^{3} / k T$. Simulated values are shown for hard-core potentials fitted to the SFA measurements converted to obtain the force [Eq. (7)] between colloidal particles of diameters $50 \mathrm{~nm}$ (open circles) and $800 \mathrm{~nm}$ (filled diamonds). Also shown is the soft-sphere potential [Eq. (1)] with a power of $n=36$ (crosses).

ther test this, and to accurately determine an effective hardsphere diameter, it is necessary to determine the phase behavior of particles with these parameters. Experimentally, hard-sphere colloidal systems have their volume fractions scaled such that the freezing volume fraction coincides with the known freezing volume fraction of the perfect hard sphere (0.494). Here we do a very similar scaling by employing Eq. (3) to set the effective hard-sphere diameter. The results are shown in Fig. 2. It can be seen that the large spheres $(800 \mathrm{~nm})$ follow the hard-sphere equation of state perfectly even at pressures above those where studies on glassy samples are typically performed. The 50-nm particles are slightly softer, and the system is able to be squashed to slightly larger densities than perfect hard spheres, at the largest pressures shown. Also shown on the graph are the results for a soft-sphere potential with $n=36$ [Eq. (1)]. Here the particles are a good deal softer, and the system is able to be compressed to higher densities at pressures around the equilibrium order-to-disorder transition. The experimental hardspheres are much harder than this. The effective hard-sphere diameters calculated using the method of Barker and Henderson [14], Eq. (3), $\sigma_{B H}$ are shown in Table I. As can be seen, they are very similar to the $\sigma_{1 k t}$ values, which makes sense for a thermal system. These values represent the separation at which the PHSA layers are just beginning to interact.

These experiments have been carried out in one solvent, decalin. In principle, we would expect there to be similar results in other compatible organic liquids, as the predominant effect is that due to the PHSA layer, rather than the solvent. To test this, we also conducted experiments in dodecane (data not shown). These give similar results, with the same hard-core distance, and a slightly softer repulsion (for example, for a 50-nm-diameter particle, $n=34$ in dodecane and 46 in decalin), which would make very little difference to the phase diagram. So the particles would be equally good hard spheres in dodecane, and it would be expected that the short-range interactions would be equally hard in other compatible solvents.

\section{CONCLUSIONS}

We have shown that colloidal hard spheres coated with PHSA have a sufficiently hard interaction that there is no deviation from the hard-sphere phase behavior up to volume fractions, in the solid phase, of at least $\phi=0.65$ (here the pressure corresponds to a glassy phase of $\phi=0.60$ ) for particles as small as $50 \mathrm{~nm}$ diameter. As particle diameter increases, the hardness of the interaction increases. Thus these particles behave as perfect hard spheres for studies of the glass transition. For experiments involving high shear rates the deviations from hard-sphere behavior could become more pronounced, however, the pair potential has now been characterized.
[1] S.E. Paulin and B.J. Ackerson, Phys. Rev. Lett. 64, 2663 (1990).

[2] W. van Megen, P.N. Pusey, and P. Bartlett, Phase Transitions 21, 207 (1990).

[3] D.W. Oxtoby, Nature (London) 413, 694 (2001).

[4] P.N. Pusey and W. van Megen, Nature (London) 320, 340 (1986)

[5] J.Z. Xue, X.L. Wu, D.J. Pine, and P.M. Chaikin, Phys. Rev. A 45, 989 (1992).

[6] K.N. Pham et al., Science 296, 104 (2002).

[7] S. Auer and D. Frenkel, Nature (London) 409, 1020 (2001).

[8] S. Auer and D. Frenkel, J. Phys.: Condens. Matter 14, 7667 (2002).

[9] U. Gasser et al., Science 292, 258 (2001).

[10] S.M. Underwood, J.R. Taylor, and W. van Megen, Langmuir 10, 3550 (1994).
[11] R. Agrawal and D.A. Kofke, Phys. Rev. Lett. 74, 122 (1995).

[12] D.A. Kofke and P.G. Bolhuis, Phys. Rev. E 59, 618 (1999).

[13] B.A.de L. Costello, P.F. Luckham, and Th.F. Tadros, Langmuir 8, 464 (1992).

[14] J.A. Barker and D. Henderson, J. Chem. Phys. 47, 4714 (1967).

[15] M. P. Allen and D. J. Tildesley, Computer Simulation of Liquids (Clarendon, Oxford, 1989).

[16] D.J. Evans and G.P. Morriss, Comput. Phys. Rep. 1, 298 (1984).

[17] J.N. Israelachvili, Intermolecular and Surface Forces (Academic Press, London, 1985).

[18] J.N. Israelachvili and G.E. Adams, J. Chem. Soc., Faraday Trans. 1 74, 975 (1978).

[19] G. Bryant et al., J. Colloid Interface Sci. 216, 401 (1999).

[20] G. Bryant et al., Langmuir (to be published). 